\title{
Quorum Sensing and Indole-3-Acetic Acid Degradation Play a Role in Colonization and Plant Growth Promotion of Arabidopsis thaliana by Burkholderia phytofirmans PsJN
}

\author{
Ana Zúñiga, ,2 María Josefina Poupin,, ${ }^{1,2}$ Raúl Donoso,, ${ }^{1,2}$ Thomas Ledger,, ${ }^{1,2}$ Nicolás Guiliani, ${ }^{3}$ \\ Rodrigo A. Gutiérrez, ${ }^{2}$ and Bernardo González ${ }^{1,2}$ \\ ${ }^{1}$ Facultad de Ingeniería y Ciencias. Universidad Adolfo Ibáñez. Santiago, Chile; ${ }^{2}$ Millennium Nucleus-PFG. FONDAP Center \\ for Genome Regulation. Pontificia Universidad Católica de Chile. Santiago, Chile; ${ }^{3}$ Departamento de Biología, Facultad de \\ Ciencias, Universidad de Chile. Santiago, Chile
}

Submitted 8 October 2012. Accepted 28 December 2012.

\begin{abstract}
Although not fully understood, molecular communication in the rhizosphere plays an important role regulating traits involved in plant-bacteria association. Burkholderia phytofirmans PsJN is a well-known plant-growth-promoting bacterium, which establishes rhizospheric and endophytic colonization in different plants. A competent colonization is essential for plant-growth-promoting effects produced by bacteria. Using appropriate mutant strains of $B$. phytofirmans, we obtained evidence for the importance of $\mathbf{N}$-acyl homoserine lactone-mediated (quorum sensing) cell-to-cell communication in efficient colonization of Arabidopsis thaliana plants and the establishment of a beneficial interaction. We also observed that bacterial degradation of the auxin indole-3-acetic acid (IAA) plays a key role in plant-growth-promoting traits and is necessary for efficient rhizosphere colonization. Wildtype $B$. phytofirmans but not the iac $C$ mutant in IAA mineralization is able to restore promotion effects in roots of $A$. thaliana in the presence of exogenously added IAA, indicating the importance of this trait for promoting primary root length. Using a transgenic $A$. thaliana line with suppressed auxin signaling (miR393) and analyzing the expression of auxin receptors in wild-type inoculated plants, we provide evidence that auxin signaling in plants is necessary for the growth promotion effects produced by $B$. phytofirmans. The interplay between ethylene and auxin signaling was also confirmed by the response of the plant to a 1-aminocyclopropane-1-carboxylate deaminase bacterial mutant strain.
\end{abstract}

The rhizosphere represents a highly dynamic space for interactions between plant roots and pathogenic and beneficial soil microorganisms (Bais et al. 2006). Nutrient availability in the rhizosphere is higher than in the bulk soil and the presence of plant exudates creates a suitable environment for growth of microorganisms (Costa et al. 2007). In the rhizosphere, molecular communication between microorganisms and their plant

Corresponding author: B. González; E-mail: bernardo.gonzalez@uai.cl; Telephone: +56-2-3311619; Fax: +56-2-3311906

* The $\boldsymbol{e}$-Xtra logo stands for "electronic extra" and indicates that three supplementary figures are published online.

(C) 2013 The American Phytopathological Society hosts plays a fundamental role in pathogenesis, and in the establishment of beneficial interactions (Mark et al. 2005).

Plant-growth-promoting rhizobacteria (PGPR) produce beneficial effects on plant growth through several mechanisms such as nitrogen fixation (Hurek et al. 2002), improved nutrient uptake (Kraiser et al. 2011), phytohormone production (Idris et al. 2007), and induction of systemic resistance (ISR) (Bakker et al. 2007). A single bacterium may possess more than one of these mechanisms (Ahmad et al. 2008). Recent studies show the importance of bacteria-to-bacteria communication in plant colonization by PGPR (Bais et al. 2004; Compant et al. 2005; Steindler et al. 2009). Bacteria can communicate by sensing and responding to small signaling molecules that make them responsive to neighboring bacteria (Whitehead et al. 2001). A variety of signaling molecules have been identified. Among them, $N$-acyl-homoserine lactones (AHL) are frequently synthesized by gram-negative bacteria that communicate through quorum sensing (QS) (Whitehead et al. 2001). Bacteria defective in QS signaling are less effective in host colonization (Bauer and Mathesius 2004; Ortíz-Castro et al. 2009; Quiñones et al. 2005), and the role of QS regulating rice growth promotion by Pseudomonas aeruginosa PUPa3 has been reported (Steindler et al. 2009).

In addition to QS molecules, other signal molecules may also play a role in plant-bacterial signaling. Among them, the auxin phytohormone indole-3-acetic acid (IAA) has been detected in culture supernatants of several rhizobacteria (Idris et al. 2007; Loper and Schroth 1986; Phi et al. 2008), suggesting that IAA may be a relevant signaling molecule in microorganisms (Bianco et al. 2006; Liu and Nester 2006; Spaepen et al. 2007, 2009; Van Puyvelde et al. 2011; Yang et al. 2007). For example, IAA triggers a broad gene-expression response in Azospirillum brasilense (Van Puyvelde et al. 2011), and IAA synthesis is controlled by a positive feedback transcriptional mechanism (Vande Broek et al. 1999). IAA has been also reported as a signaling molecule in Escherichia coli (Bianco et al. 2006), Agrobacterium tumefaciens (Liu and Nester, 2006; Yuan et al. 2008), Erwinia chrysanthemi (Yang et al. 2007), and Rhizobium etli (Spaepen et al. 2009). The production of IAA by PGPR has been involved in plant growth and root proliferation (Idris et al. 2007; Vande Broek et al. 2005). Notably, five different biosynthetic pathways and one pathway for IAA mineralization or transformation have been described in bacteria (Idris et al. 2007; Leveau and Gerards 2008).

The PGPR Burkholderia phytofirmans PsJN promotes growth of horticultural crops such as tomato, potato, and grape 
(Genin and Boucher 2004; Spaepen et al. 2007; Theocharis et al. 2012). To date, only one plant-growth-promotion molecular mechanism has been proved experimentally in $B$. phytofirmans PsJN: the reduction of the plant ethylene hormone levels by 1aminocyclopropane-1-carboxylate ACC deaminase (AcdS) (Compant et al. 2005; Vadassery et al. 2008). B. phytofirmans PsJN mutants in the acdS gene, lacking AcdS activity, are unable to promote the elongation of canola roots (Sun et al. 2009). Genome sequence analysis of B. phytofirmans PsJN shows the presence of two putative IAA synthesis pathways (the indole-3-acetamide and the tryptophan side chain oxidase pathways) and two putative QS systems associated with AHL production (Weilharter et al. 2011). In addition, it encodes one iac operon putatively involved in degradation of IAA, as reported in Pseudomonas spp. (Leveau and Gerards 2008).

In this work, we studied the importance of molecular signaling for B. phytofirmans PsJN colonization and growth promotion of Arabidopsis thaliana plants, comparing the effects of wild-type B. phytofirmans PsJN and four mutants on this plant model. We found that AHL molecular signaling is required for root colonization and plant-growth-promoting effects of this bacterium. Furthermore, we demonstrated that degradation of IAA is important in plant growth promotion by $B$. phytofirmans PsJN. The importance of plant auxin signaling for bacterial promotion of plant growth was supported by the use of an A. thaliana line with reduced expression of the auxin receptors and by analysis of the expression of genes encoding those receptors in wild-type plants inoculated with strain PsJN.

\section{RESULTS}

\section{Effect of B. phytofirmans PsJN on growth of A. thaliana.}

Based on its effects on other plant species (Ait Barka et al. 2000; Compant et al. 2005; Frommel et al. 1991; Pillay and Nowak 1997), it was supposed that B. phytofirmans PsJN would also promote growth of $A$. thaliana. Different growth parameters were evaluated in plants inoculated with strain PsJN, as well as in mock-inoculated controls. After 3 weeks of inoculation, the presence of strain PsJN increased fresh weight (FW) (169\%), primary root length $(121 \%)$, root hair number (197\%), and chlorophyll content (130\%) of A. thaliana as compared with noninoculated plants (Fig. 1A). The number of lateral roots, however, remained unchanged. AcdS has been reported as required for canola growth promotion by this bacterium (Sun et al. 2009); therefore, we tested the effect of a strain PsJN acdS mutant on A. thaliana growth. In contrast with the wild-type strain, plants inoculated with this mutant did not increase primary root length, chlorophyll content, or the number of root hairs and only partially increased FW (126\%) (Fig. 1A), indicating that AcdS activity and, therefore, ethylene levels, play a role in A. thaliana growth promotion produced by $B$. phytofirmans PsJN.

\section{Effects of $B$. phytofirmans PsJN QS mutants on growth promotion and colonization of $A$. thaliana.}

To test the role of QS in the ability of B. phytofirmans PsJN to promote plant growth, we used strain PsJN bpI.1 and bpI.2 AHL synthase mutants. In comparison with the wild-type strain, bpI.1 and bpI.2 mutants produced significantly lower levels of 3-hydroxy-C8-homoserine lactone, and the bpI.2 mutant did not produce C14-3-oxo-homoserine lactone (Supplementary Fig. S1). In addition, the bpI.1 mutant displayed a decreased swimming motility (Supplementary Fig. S2). These mutant strains grow on several carbon sources, such as fructose, 4-hydroxybenzoate, or benzoate, at the same levels as the wild-type strain (optical density of $600 \mathrm{~nm}\left[\mathrm{OD}_{600 \mathrm{~nm}}\right]=1$, after $12 \mathrm{~h}$ of culture), indicating that their general metabolism is not affected. Concerning turnover of IAA (see next section), the bpI.1 and bpI.2 mutants synthesized IAA (19.6 and 19.9 $\mu \mathrm{g} / \mathrm{ml}$, respectively) to an amount similar to the wild-type strain $(20.0 \mu \mathrm{g} / \mathrm{ml})$, and the three strains grow with $2.5 \mathrm{mM}$ IAA as the sole carbon and energy source at the same level: $\mathrm{OD}_{600 \mathrm{~nm}}=$ 0.6 , after $26 \mathrm{~h}$ of growth. After 3 weeks of inoculation, the $B$. phytofirmans PsJN bpI.1 mutant was unable to increase primary root length (89\%), FW (87\%), and chlorophyll content ( $82 \%$ ) of $A$. thaliana, in contrast with the wild-type strain (Fig. 1A). Only the number of root hairs increased (150\%) in plants inoculated with this mutant, although to a lower extent compared with the wild-type strain. In contrast, plants inoculated with the bpI.2 mutant increased primary root length, number of roots hairs, chlorophyll content, and number of lateral roots to an extent similar to that of plants inoculated with the wildtype strain (Fig. 1A).

To determine whether differences in growth promotion of $A$. thaliana by $B$. phytofirmans PsJN and the bpI.1 mutant were due to different capabilities for rhizospheric and endophytic colonization, bacterial colonization of this plant was evaluated in gnotobiotic culture systems. Strain PsJN bpI.1 mutant showed a reduced ability for rhizospheric colonization of $A$. thaliana ( $8 \pm 0.1 \log \mathrm{CFU} / \mathrm{mg} \mathrm{FW})$ compared with the wildtype strain and the $b p I .2$ mutant $(9.84 \pm 0.01$ and $9 \pm 0.3 \log$ CFU/mg FW, respectively). Epifluorescence and confocal
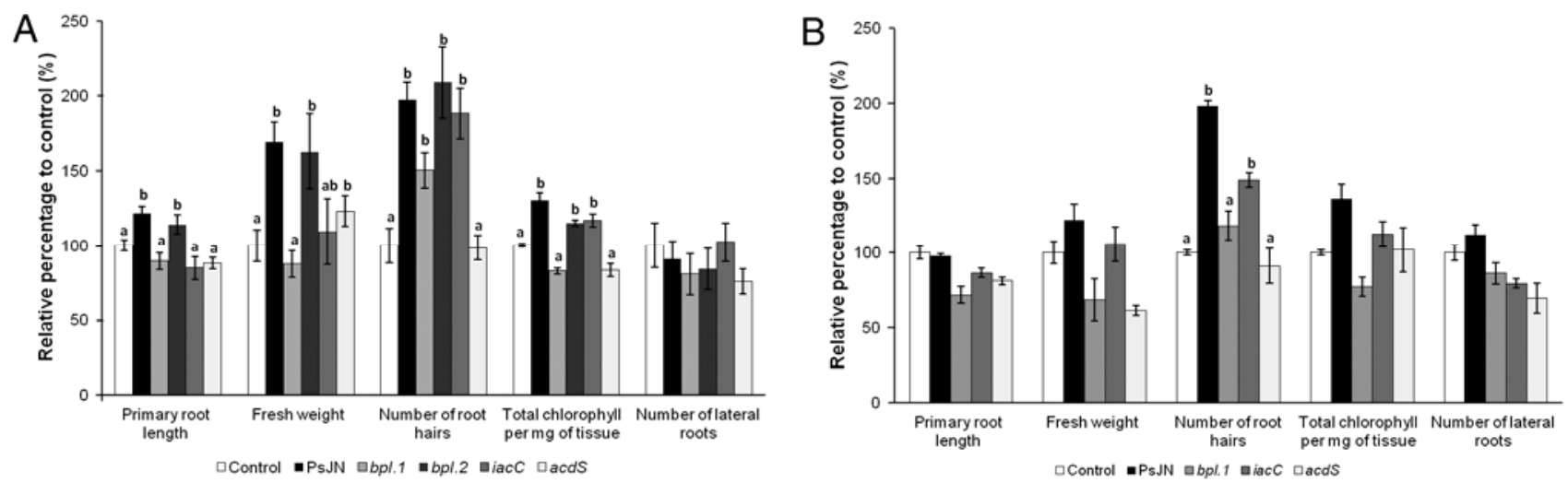

Fig. 1. Plant growth and metabolic parameters in A, gnotobiotic Arabidopsis thaliana col-0 and B, A. thaliana miR393 overexpressor lines, culture systems inoculated with Burkholderia phytofirmans PsJN, its bpI.1, bpI.2, iacC, and acdS mutants, or noninoculated (control). Growth parameters were measured 3 weeks after inoculation. Bars show mean percentage values with respect to control plants, and the error bars indicate standard deviations from average of three biological replicate experiments for each treatment. Different letters indicate statistically significant differences between treatments (one-way analysis of variance Tukey's honestly significant difference tests, $P<0.05$ ). 
microscopy analysis of $A$. thaliana roots inoculated with the wild-type PsJN or the $b p I .1$ mutant green fluorescent protein (GFP)-tagged strains showed green fluorescent bacterial cells mainly attached to lateral root emergences and root tips and highly spread on hair roots; however, strong adherence to tissues was observed only with the wild-type strain (Supplementary Fig. S3) and the bpI.2 mutant (data not shown), whereas the bpI.1 mutant cells are present but seem to move loosely on the root surface. Endophytic colonization of $A$. thaliana by both bpI. 1 and bpI. 2 mutant strains was statistically lower $(5 \pm 0.9$ and $4.9 \pm 0.2 \log \mathrm{CFU} / \mathrm{mg} \mathrm{FW}$, respectively) than with the wildtype strain $(7.9 \pm 0.5 \log \mathrm{CFU} / \mathrm{mg} \mathrm{FW})$. These results indicate that QS signaling is required for rhizospheric and endophytic colonization of strain PsJN in A. thaliana roots.

\section{Effect of an IAA degradation mutant of $B$. phytofirmans on growth promotion and colonization of $A$. thaliana.}

To study whether IAA degradation by $B$. phytofirmans PsJN has a role on plant growth promotion, the mutant $i a c C$, completely unable to grow on IAA because it lacks a functional aromatic ring hydroxylating dioxygenase involved in the IAA degradation pathway (R. A. Donoso, unpublished results), was constructed and analyzed. This mutant grows on different carbon sources such as fructose, 4-hydroxybenzoate, or benzoate at the same yields and rates as the wild-type strain, and produces normal extracellular levels of IAA $(18 \mu \mathrm{g} / \mathrm{ml})$. After 3 weeks of inoculation of a gnotobiotic A. thaliana culture with the iac $C$ mutant, no differences were observed in number of root hairs, chlorophyll content, and number of lateral roots with respect to plants inoculated with the wild-type PsJN (Fig. 1A). However, the growth promotion effects of the wild-type strain on FW and primary root length were not observed with the iacC mutant (Fig. 1A), indicating that degradation of IAA by $B$. phytofirmans PsJN is required for full plant growth promotion. Neither the rhizospheric $(9.4 \pm 0.1$ versus $9.84 \pm 0.01$ $\log \mathrm{CFU} / \mathrm{mg} \mathrm{FW})$ nor the endophytic $(6.8 \pm 0.6$ versus $7.9 \pm$ $0.5 \log$ CFU/mg FW) colonization levels in A. thaliana were affected in this mutant compared with the wild-type PsJN. To further study the effect of IAA degradation on growth promotion by $B$. phytofirmans, the abilities of the wild-type strain and iac $C$ mutant to abolish the effects of exogenously added IAA on root development were compared. Two concentrations of IAA were exogenously added when seed were placed on plates. A sharp decrease in primary root lengths was verified at $1 \mu \mathrm{M}$ IAA (Fig. 2A and B). The inoculation with the wild-type strain completely prevented the primary root length inhibition effect of such concentration of IAA, whereas the iacC mutant only partially reverted such inhibition (Fig. 2A and B).

\section{Effect of B. phytofirmans PsJN and its mutants in growth promotion of a transgenic $A$. thaliana with reduced auxin signaling.}

To determine whether IAA signaling in the plant is required for plant growth promotion by B. phytofirmans PsJN, a transgenic line of $A$. thaliana overexpressing miR393 (ox-miR393) was used. These plants have reduced expression of the auxin receptor genes TIR1, AFB1, AFB2, and AFB3 and reduced auxin signaling (Navarro et al. 2006). Gnotobiotic cultures of ox-miR393 plants were inoculated with the wild-type PsJN strain or the $i a c C, b p I .1$, and $a c d S$ mutants. Three weeks after the inoculation, the wild-type strain and some of its mutants failed to produce an increase in primary root length, FW, or total chlorophyll content, as seen in wild-type plants (Fig. 1B). Only the number of root hairs increased in the transgenic plant inoculated with the wild-type strain and the iacC mutant (Fig. 1B). B. phytofirmans colonized the rhizosphere of gnotobiotic cultures of ox-miR393 plants at normal levels $(9.8 \pm 0.1 \mathrm{log}$ $\mathrm{CFU} / \mathrm{mg} \mathrm{FW}$ ). However, no B. phytofirmans endophytes were found in ox-miR393 plants. The transcript levels of the auxin receptor genes TIR1, AFB1, AFB2, and AFB3 were analyzed in wild-type plants inoculated with strain PsJN using quantitative real time polymerase chain reaction (qRT-PCR) (Fig. 3). Inoculation did not affect the expression of these genes in plants of four leaves (13 days after sowing). However, AFB1 and AFB3 genes were up regulated in PsJN-inoculated plants at the stage of six leaves (19 days after sowing).

\section{DISCUSSION}

B. phytofirmans colonizes the rhizosphere and internal tissues of its plant hosts (Compant et al. 2005; Sessitsch et al. 2005). In this work, we showed that the plant model A. thaliana may also be colonized by strain PsJN. We also found that AcdS activity and QS are required for plant growth promotion and rhizosphere and endophytic colonization in A. thaliana. We report, for the first time, the involvement of IAA in plant growth promotion by this well-known PGPR. An IAA degradation mutant is not effective in promoting plant growth and in preventing adverse effects of exogenously added IAA; the plant growth promotion effects are partially abolished in $A$. thaliana plants with reduced IAA
A

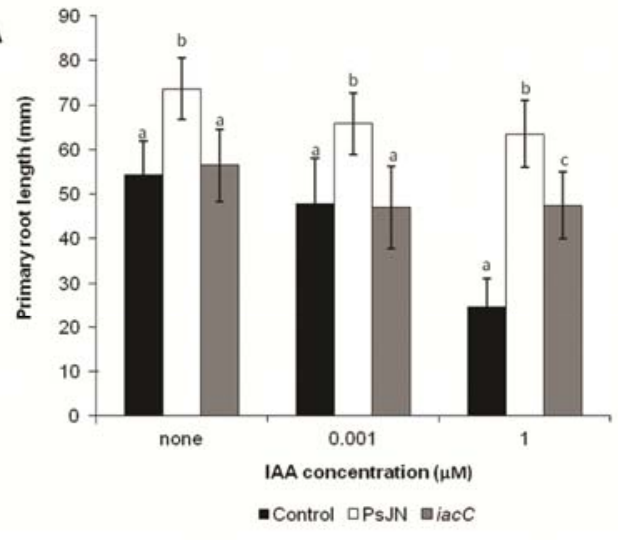

B

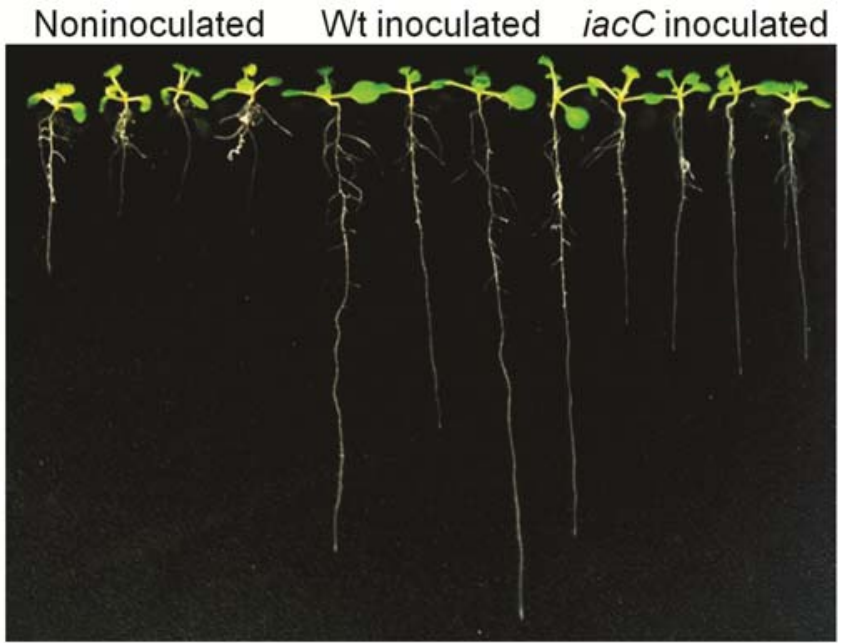

Fig. 2. A, Effect of exogenous addition of indole-3-acetic acid (IAA) on the elongation of primary roots in plants of Arabidopsis thaliana inoculated with Burkholderia phytofirmans PsJN or its iacC mutant. Different letters indicate statistically significant differences between root lengths (one-way analysis of variance Tukey's honestly significant difference tests, $P<0.05$ ). B, Photograph of 10-day-old seedlings grown exposed to $1 \mu \mathrm{M}$ exogenous IAA. 
signaling, and the expression of some auxin receptors is upregulated in inoculated plants. In this context, it is worth mentioning that batch cultures of $B$. phytofirmans degrading IAA present twofold longer lag phases compared with other aromatic carbon sources (data not shown), and the synthesis of IAA by B. phytofirmans occurs before IAA degradation, suggesting that IAA turnover is carefully controlled in this bacterium (R. A. Donoso, unpublished results). Furthermore, qRT-PCR detection of iacC gene transcripts and $i a c C$ gene promoter transcriptional fusion analysis clearly demonstrated that IAA regulates its degradation and is able to significantly induce iacC gene expression (R. A. Donoso, unpublished results).

Although some hints of the mechanism or mechanisms used by $B$. phytofirmans to promote plants growth have been proposed (Ait Barka et al. 2000; Sessitsch et al. 2005), thus far, only the involvement of AcdS in primary root length stimulation in canola had been described (Sun et al. 2009). In our model plant (Fig. 1), the effect of PsJN inoculation on root elongation is certainly lesser than the elongation observed with other plant models such as canola (Sun et al. 2009); however, we have shown it to be reproducible and statistically significant, which allows us to differentiate the effects of the wildtype strain and the $a c d S$ mutant strain. Interestingly, tests with the IAA degradation mutant strain ( $\mathrm{iacC}$ ) also showed no improvement of primary root lengths. It has been described that root growth can be stimulated or inhibited depending on the concentration of IAA produced by bacteria (López-Bucio et al. 2007; Persello-Cartieux et al. 2001). In plants inoculated with the iacC mutant, IAA levels in roots should be higher than in roots inoculated with the wild-type PsJN strain, because this mutant cannot degrade IAA and, thus, the plant would be more susceptible to primary root shortening. Leveau and Lindow (2005) have described similar effects in radish plants inoculated with $P$. putida 1290 , a strain that catabolizes IAA. This can be explained through the cross-talk between auxin and ethylene synthesis in plants, where auxin increases ACC synthase gene transcription, stimulating ethylene synthesis and, conversely, ethylene promotes expression of IAA biosynthetic genes increasing IAA levels at the root tip (Růzicka et al. 2007; Strader et al. 2010; Swarup et al. 2007). Therefore, high concentrations of IAA lead to high levels of ethylene, decreasing root cell length (Strader et al. 2010). Consistently, we found that strain PsJN abolishes the effect of exogenous IAA that decreases primary root length and that A. thaliana plants inoculated with the iacC mutant showed only a partial reversion of the effect of exogenous IAA. Although we cannot discard the possibility that the iacC mutant partially transforms exogenously added IAA, this partial effect may be also explained through cross talk with the AcdS effect, which is still operative in the iacC mutant, that reduces levels of ethylene and stimulates root elongation. These observations suggest that both AcdS and IAA degradation activities are important in primary root growth promotion by $B$. phytofirmans PsJN.

The use of an $A$. thaliana transgenic line overexpressing miR393 provided additional support for the involvement of plant IAA signaling in plant growth promotion by $B$. phytofirmans PsJN. The positive effects of bacterial inoculation almost completely disappeared in ox-mi393 plants. Although rhizospheric colonization still takes place in this transgenic line, endophytic colonization by $B$. phytofirmans was grossly impaired. Similar results were found with ox-miR393 plants inoculated with pathogen $P$. syringae DC 3000 , where repression of auxin signaling restricted its growth inside plants (Navarro et al. 2006). The results obtained with this A. thaliana transgenic line which, among other effects in auxin signaling, has reduced expression of auxin receptor genes (Navarro et al. 2006), prompted us to test the effect of inoculation with strain PsJN on the expression of these genes, finding upregulation of the expression of AFB1 and AFB3 genes in a developmental timecontrolled manner (Fig. 3). Furthermore, our lab has analyzed recently global transcription changes in Arabidopsis plants inoculated with strain PsJN, finding that genes implicated in auxin pathway were significantly regulated (M. J. Poupin and T. Timmermann unpublished results).

Bacterial IAA synthesis has been proposed as an important feature in pathogenic bacteria-plant interactions (Comai and
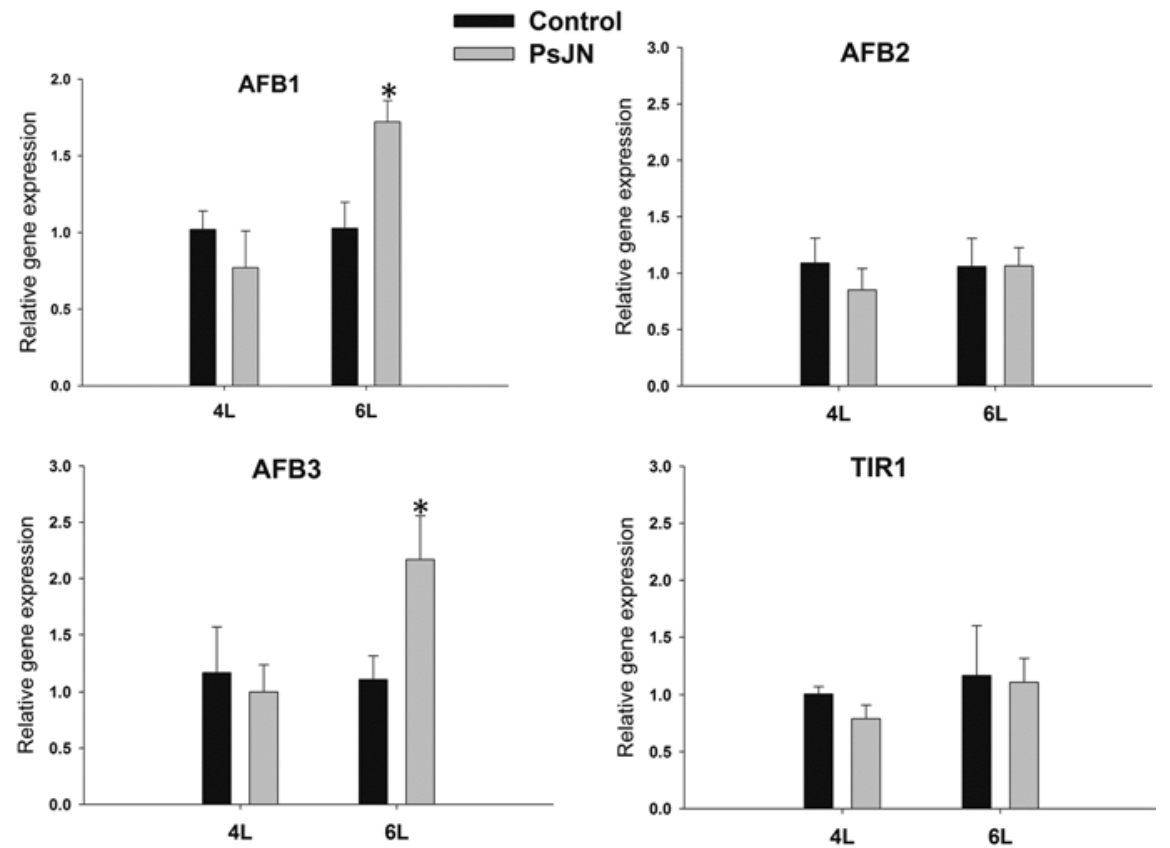

Fig. 3. Effect of PsJN inoculation in the gene expression of auxin receptors in wild-type plants. Quantitative real-time polymerase chain reaction determinations of relative levels of gene expression in complete plants at four rosette leaves $(4 \mathrm{~L})$ or six rosette leaves $(6 \mathrm{~L})$ stages. Data are means \pm standard errors of three biological replicates. Asterisk indicates statistical significance (AFB1: Mann-Whitney $\mathrm{U}$ test, $Z=-1,963, P<0.05$; AFB3: Mann-Whitney $\mathrm{U}$ test, $Z=-1,727, P<0.05)$. 
Kosuge 1982; Navarro et al. 2006) as well as in phytostimulation processes (Patten and Glick 2002a and b) and, consequently, should play a relevant role in plant growth promotion mechanisms in B. phytofirmans and other PGPR. The results reported here support that possibility; unfortunately, the construction of IAA synthesis strain PsJN mutants, which may allow testing the effects on plant growth promotion, is not simple because this strain possesses at least two putative IAA biosynthetic pathways: the indole-3-acetamide pathway and the tryptophan side chain oxidase pathway (Weilharter et al. 2011). In addition, we carried out homology searches, in the strain PsJN genome, of additional IAA synthesis pathways and we found putative marker genes for the indole-3-pyruvate, indole-3-acetonitrile, and tryptamine pathway. We have generated single mutants for all these marker genes but these mutants still synthesize IAA (R. A. Donoso, unpublished results), indicating that strain PsJN carries multiple pathways to synthesize this compound. This is similar to the case of Azospirillum brasilense, in which at least three different pathways of IAA synthesis are functional (Prinsen et al. 1993).

Other molecular signaling processes may contribute to PGPR performance. Recently, it has been described that the PGPR $A$. lipoferum cell-to-cell communication QS system mediated by AHL is implicated in rhizosphere competence and adaptation to plant roots (Boyer et al. 2008). Furthermore, a QS system has been involved in the regulation of plant-growth-promoting traits of $P$. aeruginosa PUPa3 (Steindler et al. 2009). We reported here that a bpI.1 mutant in the QS system of B. phytofirmans, which produces lower AHL levels than the wild-type strain, is unable to promote growth on Arabidopsis thaliana. This is probably due to the lower rhizosphere colonization and null endophytic colonization ability of the mutant compared with the wild-type strain. Consistently, less biofilm formation on plates fed with root exudates (A. Zúñiga, unpublished results), low motility, and grossly impaired adherence to A. thaliana root surface are observed in the bpI.1 mutant compared with the wild-type strain. Furthermore, analysis of transcript levels of genes involved in swimming motility (Bphyt_3794, Bphyt_3820, Bphy_3804, and Bphyt_3770) and exopolysaccharide production (Bphyt_4056, Bphyt_6264, Bphyt_0818, and Bphyt-1955) showed, for most of them, downregulated or repressed expression in the PsJN bpI.1 mutant compared with expression in wild-type PsJN (A. Zúñiga, unpublished results). Motility, attachment to roots, and biofilm formation are important traits for root surface colonization (Danhorn and Fuqua 2007; Steindler et al. 2009), because PGPR have been reported to colonize developing small biofilms along epidermal fissures (Ramey et al. 2004). Regulation by a QS system may influence key steps leading to biofilm formation required for plant growth promotion. This may explain low rhizosphere and endophytic colonization and lack of plant-growthpromoting traits observed with the PsJN bpI.l mutant, and low endophytic levels of the bpI.2 mutant. The production of exopolysaccharide polymer has been reported in strain PsJN and in other plant-associated Burkholderia spp., and it is believed to be involved in the plant-bacterium interaction (Ferreira et al. 2010). Interestingly, impaired exopolysaccharide production affected endophytic colonization by a QS mutant in B. kururiensis (Suárez-Moreno et al. 2010). Although the production of cell-wall-degrading endoglucanases by strain PsJN plays a role in grapevine endophytic colonization (Compant et al. 2005), both $b p I$ mutants and the wild-type PsJN strain showed no differences in endoglucanase levels (data not shown), indicating that lower endophytic colonization found with A. thaliana is not due to the lack of this cell-wall-degrading activity. Additional studies with QS mutants are required for full understanding of the role of a QS system in endophytic and rhizospheric colonization and, ultimately, plant growth promotion.

\section{MATERIALS AND METHODS}

\section{Bacterial strains and growth conditions.}

B. phytofirmans PsJN was obtained from A. Sessitsch. Wildtype PsJN and the four mutants were grown at $30^{\circ} \mathrm{C}$ on Dorn mineral salts medium (Dorn et al. 1974) containing $10 \mathrm{mM}$ fructose, $5 \mathrm{mM}$ 4-hydroxybenzoate or benzoate, or $2.5 \mathrm{mM}$ IAA as the sole carbon and energy source and, if required, kanamycin $\left(50 \mu \mathrm{g} \mathrm{ml}^{-1}\right)$ or spectinomycin $\left(100 \mu \mathrm{g} \mathrm{ml}^{-1}\right)$ for $12 \mathrm{~h}$. For growth tests of strains on $2.5 \mathrm{mM}$ IAA, cells were grown for $36 \mathrm{~h}$, biomass measured at $\mathrm{OD}_{600 \mathrm{~nm}}$, and three replicates were performed for each growth measurement.

\section{Construction of B. phytofirmans PsJN mutants.}

Internal fragments of the bpI.1 gene (locus Bphyt_0126, AHL synthase of chromosome 1 QS system), bpI.2 gene (locus Bphyt_4275, AHL synthase of chromosome 2 QS system), iacC gene (locus Bphyt_2156, aromatic ring hydroxylating dioxygenase involved in catabolism of IAA), and acdS gene (locus Bphyt_5397, 1-aminocyclopropane-1-carboxylic acid deaminase) sequences were amplified by PCR, using the primer pairs bpi.1mutFW (GACGGAGGCCAGCAATATAA) and bpi.1mutRV (GTATGGGAGATGTCGCGATT), bpI.2mutFW (GA ACGTCACCAGTTCGTGAAT) and bpI.2mutRV (ATGGAGA TCGACGGCTATGA), iacCmutFW (GGTCAACGTCTTGCA GAACC) and iacCmutRV (GTTTCGTCGTCGATCGATTT), and acdSmutFW (CGAATATCTGATCCCCGAAG) and acdSmutRV (AAGCCGATGTCGAAACCAT), respectively. The PCR products were cloned using the pCR2.1-TOPO system (Invitrogen, Carlsbad, CA, U.S.A.) to generate plasmids pCR2.1bpi.1, pCR2.1iacC, and pCR2.1acdS. The bpI.2 PCR product was cloned using the pCR8/GW/TOPO system (Invitrogen), to generate plasmid pCR8bpI.2. These plasmids were electroporated in B. phytofirmans PsJN to get one recombination event disruption of the target gene, and recombinants were selected on Luria-Bertani (LB) agar containing kanamycin at $50 \mu \mathrm{g} / \mathrm{ml}$ to obtain strain PsJN bpi.l, strain PsJN iacC, and PsJN acdS mutants and spectinomycin $100 \mu \mathrm{g} / \mathrm{ml}$ to obtain strain PsJN bpI.2 mutant. Correct insertions in mutant strains were confirmed by PCR and sequencing.

\section{RNA extraction, cDNA synthesis, and qRT-PCR analysis.}

For RNA extraction, plants of LP.04 stage (Boyes et al. 2001) (four rosette leaves visible, corresponding to 13 days after sowing) or LP.06 stage (Boyes et al. 2001) (six rosette leaves visible, corresponding to 19 days after sowing) were used. Ten plantlets for each treatment were collected in liquid nitrogen and ground with a pestle in an Eppendorf tube. Then, RNA was obtained using the Trizol method following the manufacturer's instructions (Invitrogen). For cDNA synthesis, $1 \mu \mathrm{g}$ of total RNA treated with DNAse I (RQ1; Promega Corp., Madison, WI, U.S.A.) was reverse transcribed with random hexamer primers using the Improm II reverse transcriptase (Promega Corp.), according to the manufacturer's instructions. RT-PCR was performed using the Brilliant SYBR Green QPCR Master Reagent Kit (Agilent Technologies, Santa Clara, CA, U.S.A.) and the Eco Real-Time PCR detection system (Illumina, San Diego, CA, U.S.A.). The PCR mixture $(15 \mu \mathrm{l})$ contained $2.0 \mu \mathrm{l}$ of template cDNA (diluted 1:10) and $140 \mathrm{nM}$ each primer. Amplification was performed under the following conditions: $95^{\circ} \mathrm{C}$ for $10 \mathrm{~min}$; followed by 40 cycles of $94^{\circ} \mathrm{C}$ for $30 \mathrm{~s}, 57^{\circ} \mathrm{C}$ for $30 \mathrm{~s}$, and $72^{\circ} \mathrm{C}$ for $30 \mathrm{~s}$; followed by a melting cycle from 55 to $95^{\circ} \mathrm{C}$. Relative gene expression calculations were conducted as described in the software manufacturer's instructions: an accurate ratio between the expression of the gene of interest (GOI) and the housekeeping (HK) gene was calculated according to the following $2^{-(\Delta \mathrm{CtGOI}-\mathrm{HK})}$. Then, 
gene expression levels were normalized to the average value of the expressions in the control treatment. AtSAND (AT2G28390) was used as the $\mathrm{HK}$ gene using previously described primer pairs (Czechowski et al. 2005). Primer pairs for TIR1 (AT3G62980), AFB1 (AT4G03190), AFB2 (AT3G26810), and AFB3 (AT1G12820) have been used elsewhere (Vidal et al. 2010). All experiments were performed in three biological and two technical replicates.

\section{Green fluorescence protein labeling.}

Strain PsJN and its mutants were tagged with the GFP marker gene using a mini-Tn5 system (Mathysse et al. 1996), which forms stable genomic insertions. Wild-type PsJN and PsJN bpI.1 and PsJN bpI.2 mutants were conjugated with Escherichia coli PRK2073, as helper in a triparental mating, and E. coli S17, which contains the plasmid with miniTn5GFP construct carrying tetracycline resistance. Transconjugants carrying the GFP marker were selected on LB containing tetracycline at $10 \mu \mathrm{g} / \mathrm{ml}$. GFP-labeled cells were examined by an Optical Fluorescence microscope in Model Nikon Eclipse 50i (Nikon, Tokyo) equipped with GFP HYQ and $\mathrm{G}-2 \mathrm{E} / \mathrm{C}$ filters.

\section{Measurement of IAA synthesis.}

Bacterial cells were grown with $5 \mathrm{mM}$ fructose at $\mathrm{OD}_{600 \mathrm{~mm}}=$ 1, which corresponds to the logarithmic phase of growth. ATtR this point, bacterial cells were incubated with $2.5 \mathrm{mM}$ tryptophan (as inducer of IAA synthesis) for $3 \mathrm{~h}$; then, IAA synthesis was measured on supernatants of cultures using Salkowski reagent, as described previously (Glickmann and Dessaux 1995). Three replicates were performed for each IAA synthesis measurement.

\section{Preparation of bacterial inoculants.}

For plant colonization and growth effect experiments, $B$. phytofirmans PsJN and its four mutant derivative strains (PsJN bpI.1, PsJN bpI.2, PsJN acdS, and PsJN iacC) were routinely grown in Dorn mineral salts medium (Dorn et al. 1974) with $2 \mathrm{mM}$ fructose in an orbital shaker $(150 \mathrm{rpm})$ for $24 \mathrm{~h}$ at $30^{\circ} \mathrm{C}$. Cell suspensions from each inoculum were then obtained and adjusted to approximately $10^{8} \mathrm{CFU} / \mathrm{ml}$, as determined by plate counting. Then, each strain at $10^{4} \mathrm{CFU} / \mathrm{ml}$ was homogeneously inoculated on $1 \%$ agar plates containing Murashige and Skoog (MS) basal salt mixture (Sigma-Aldrich, St. Louis).

\section{Plant growth.}

A. thaliana ecotype Col-0 and the A. thaliana transgenic line ox-miR393 (Navarro et al. 2006; Vidal et al. 2010) were used. Seed were surface sterilized with $50 \%$ (vol/vol) commercial chlorine bleach for $7 \mathrm{~min}$ and washed three times in sterile distilled water. Then, seed were kept at $4^{\circ} \mathrm{C}$ for 2 days in the absence of light to produce stratification. After that, seed were sown in sterile plastic petri dishes with $1 \%$ agar plates containing MS basal salt mixture (Sigma-Aldrich) inoculated or not with bacteria. Eight seeds were sown in each plate and six plates were used for each treatment: control without bacteria, wild type, and $b p I .1, b p I .2, i a c C$, and $a c d S$ mutant strains. To perform exogenous IAA degradation assays, seed were germinated and grown for 2 weeks on plates with MS medium containing 0.001 or $1 \mu \mathrm{M}$ IAA inoculated or not with either wild-type or iacC mutant strains. Plates were placed vertically, sealed with parafilm, and arranged in a completely randomized design. The plant growth chamber was run with a cycle of $12 \mathrm{~h}$ of light and $12 \mathrm{~h}$ of darkness and a temperature of $22 \pm 2{ }^{\circ} \mathrm{C}$. Three biological replicates were carried out.

\section{Determination of rhizospheric and endophytic bacterial colonization.}

For rhizospheric colonization tests, 3 -week-old plants were removed from inoculated MS media agar and washed in phosphate buffer solution, with vortex agitation. Extracted liquid material was serially diluted with Dorn mineral salts medium before plating on Dorn medium plates supplemented with benzoate as the sole carbon and energy source. The CFU/mg $\mathrm{FW}$ was determined after $48 \mathrm{~h}$ of incubation at $30^{\circ} \mathrm{C}$. For endophytic colonization tests, 3-week-old plantlets inoculated with GFP-labeled PsJN strains were removed from the agar plates, surface sterilized with $70 \%$ ethanol for $1 \mathrm{~min}$ followed by $1 \%$ commercial chlorine bleach and a $0.01 \%$ Tween 20 solution for $1 \mathrm{~min}$, then washed three times in sterile distilled water (adapted from Compant and associates [2005]). Plating the distilled water from a final wash on R2A medium routinely controlled sterility on these plants. Then, the sterilized plant material was macerated in sterile mortars and the disrupted tissue was resuspended in $1 \mathrm{ml}$ of sterile $50 \mathrm{mM}$ phosphate buffer to obtain an aqueous extract. CFU/mg FW was determined by serial dilutions of these extracts in R2A agar plates after $48 \mathrm{~h}$ of incubation at $30^{\circ} \mathrm{C}$ and plates were examined under UV light using an Optical Epi-fluorescence Nikon Eclipse 50i microscope (Nikon). Experiments were conducted with six plants analyzed for each treatment, in two biological replicates.

\section{Microscopy analyses.}

To determine rhizosphere colonization by GFP-marked strains, treated and untreated plant root surfaces were examined by optical epifluorescence and confocal microscopy. Epifluorescence images were taken using a Nikon Eclipse 50i microscope (Nikon) equipped with GFP HYQ and G2E/C filters, and photographs were taken with a DS Fil digital camera (Nikon). Confocal microscope images were obtained using Olympus FluoView 1000 confocal laser scanning (Olympus, Tokyo) equipped with high-performance sputtered filters.

\section{Determination of the plant growth parameters.}

In all, 25 plantlets from each inoculated treatment as well as 25 noninoculated plantlets were analyzed. Root length and lateral roots number were measured directly in harvested plants, and FW was recorded as previously described (Compant et al. 2005; Nowak et al. 1995; Sessitsch et al. 2005). The chlorophyll contents were also determined following a published procedure (Porra et al. 1989). Chlorophyll was extracted from leaves of A. thaliana in N,N-9dimethylformamide for $24 \mathrm{~h}$ at $4^{\circ} \mathrm{C}$ in the dark, and chlorophyll a and chlorophyll b concentrations were measured simultaneously by spectrophotometry. For root hair number measurements, segments at a distance of $1 \mathrm{~cm}$ from the root tip of the primary root were analyzed using light microscopy.

\section{Statistical analysis.}

Data for plant growth parameters and population density ( $\mathrm{CFU} / \mathrm{mg} \mathrm{FW}$ ) were statistically analyzed using one-way analysis of variance. The chlorophyll contents and CFU/mg FW were subjected to logarithmic transformation before data analysis. When analysis of variance showed significant treatment effects, Tukey's honestly significant difference $(P<0.05)$ test was applied to make comparisons between treatments. Statistical analyses of plant gene expression were performed using the Mann-Whitney Test for nonparametrics data in the statistical software package STATISTICA (version 6.0; StatSoft Inc., Tulsa, OK, U.S.A.). 


\section{ACKNOWLEDGMENTS}

We thank J. Jones for providing the A. thaliana miR393 overexpressor lines and G. León from Universidad Andres Bello for technical and interpretation advice in confocal microscopy. This work was funded by the FONDECYT grants 3100040 and 3090051, the Millennium Nuclei in "Microbial Ecology and Environmental Microbiology and Biotechnology" grant P/04-007-F, and "Plant Functional Genomics" grant P/06-009-F. Additional support from CONICYT grant 79090016 is acknowledged. A. Zúñiga and R. Donoso are CONICYT-PhD fellows.

\section{LITERATURE CITED}

Ahmad, F., Ahmad, I., and Khan, M. S. 2008. Screening of free-living rhizospheric bacteria for their multiple plant growth promoting activities. Microbiol. Res. 163:173-181.

Ait Barka, E., Belarbi, A., Hachet, C., Nowak, J., and Audran, J.-C. 2000. Enhancement of in vitro growth and resistance to gray mould of Vitis vinifera co-cultured with plant growth-promoting rhizobacteria. FEMS (Fed. Eur. Microbiol. Soc.) Microbiol. Lett. 186:91-95.

Bais, H. P., Park, S. W., Weir, T. L., Callaway, R. M., and Vivanco, J. M. 2004. How plants communicate using the underground information superhighway. Trends Plant Sci. 9:26-32.

Bais, H. P., Weir, T. L., Perry, L. G., Gilroy, S., and Vivanco, J. M. 2006. The role of root exudates in rhizosphere interactions with plants and other organisms. Annu. Rev. Plant Biol. 57:233-266.

Bakker, P. A., Pieterse, C. M., and van Loon, L. C. 2007. Induced systemic resistance by fluorescent Pseudomonas spp. Phytopathology 97:239-243.

Bauer, W. D., and Mathesius, U. 2004. Plant responses to bacterial quorum sensing signals. Curr. Opin. Plant Biol. 7:429-433.

Bianco, C., Imperlini, E., Calogero, R., Senatore, B., Amoresano, A., Carpentieri, A., Pucci, P., and Defez, R. 2006. Indole-3-acetic acid improves Escherichia coli's defenses to stress. Arch. Microbiol. 185:373-382.

Boyer, M., Bally, R., Perrotto, S., Chaintreuil, C., and Wisniewski-Dye, F. 2008. A quorum-quenching approach to identify quorum sensing-regulated functions in Azospirillum lipoferum. Res. Microbiol. 159:699-708.

Boyes, D. C., Zayed, A. M., Ascenzi, R., McCaskill, A. J., Hoffman, N. E., Davis, K. R., and Gorlach, J. 2001. Growth stage-based phenotypic analysis of Arabidopsis: A model for high throughput functional genomics in plants. Plant Cell 13:1499-1510.

Comai, L., and Kosuge, T. 1982. Cloning characterization of iaaM, a virulence determinant of Pseudomonas savastanoi. J. Bacteriol. 149:40-46.

Compant, S., Reiter, B., Sessitsch, A., Nowak, J., Clement, C., and Ait Barka, E. 2005. Endophytic colonization of Vitis vinifera L. by a plant growth-promoting bacterium, Burkholderia sp. strain PsJN. Appl. Environ. Microbiol. 71:1685-1693.

Costa, R., Gomes, N. C. M., Krögerrecklenfort, E., Opelt, K., Berg, G., and Smalla, K. 2007. Pseudomonas community structure and antagonistic potential in the rhizosphere: Insights gained by combining phylogenetic and functional gene-based analyses. Environ. Microbiol. 9:2260-2263.

Czechowski, T., Stitt, M., Altmann, T., Udvardi, M. K., and Scheible, W. R. 2005. Genome-wide identification and testing of superior reference genes for transcript normalization in Arabidopsis. Plant Physiol. 139:517.

Danhorn, T., and Fuqua, C. 2007. Biofilm formation by plant-associated bacteria. Annu. Rev. Microbiol. 61:401-422.

Dorn, E., Hellwig, M., Reineke, W., and Knackmuss, H.-J. 1974. Isolation and characterization of a 3-chlorobenzoate degrading pseudomonad. Arch. Microbiol. 99:61-70.

Ferreira, A., Leitao, J., Silva, I., Pinheiro, P., Sousa, S., Ramos, C., and Moreira, L. 2010. Distribution of cepacian biosynthesis genes among environmental and clinical Burkholderia strains and role of cepacian exopolysaccharide in resistance to stress conditions. Appl. Environ. Microbiol. 76:441-450.

Frommel, M. I., Nowak, J., and Lazarovits, G. 1991. Growth enhancement and developmental modifications of in vitro grown potato (Solanum tuberosum ssp. tuberosum) as affected by a nonfluorescent Pseudomonas sp. Plant Physiol. 96:928-936.

Genin, S., and Boucher, C. 2004. Lessons learned from the genome analysis of Ralstonia solanacearum. Annu. Rev. Phytopathol. 42:107-134.

Glickmann, E., and Dessaux, Y. 1995. A critical examination of the specificity of the Salkowski reagent for indolic compounds produced by phytopathogenic bacteria. Appl. Environ. Microbiol. 61:793-796.

Hurek, T., Handley, L. L., Reinhold-Hurek, B., and Piché, Y. 2002. Azoarcus grass endophytes contribute fixed nitrogen to the plant in an unculturable state. Mol. Plant-Microbe Interact. 15:233-242.
Idris, E. E., Iglesias, D. J., Talon, M., and Borriss, R. 2007. Tryptophandependent production of indole-3-acetic acid (IAA) affects level of plant growth promotion by Bacillus amyloliquefaciens FZB42. Mol. Plant-Microbe Interact. 20:619-626.

Kraiser, T., Gras, D. E., Gutiérrez, A. G., González, B., and Gutiérrez, R. A. 2011. A holistic view of nitrogen acquisition in plants. J. Exp. Bot. 62:1455-1466.

Leveau, J. H., and Gerards, S. 2008. Discovery of a bacterial gene cluster for catabolism of the plant hormone indole 3-acetic acid. FEMS (Fed. Eur. Microbiol. Soc.) Microbiol. Ecol. 65:238-250.

Leveau, J. H., and Lindow, S. E. 2005. Utilization of the plant hormone indole-3-acetic acid for growth by Pseudomonas putida strain 1290. Appl. Environ. Microbiol. 75:2365-2371.

Liu, P., and Nester, E. W. 2006. Indoleacetic acid, a product of transferred DNA, inhibits vir gene expression and growth of Agrobacterium tumefaciens C58. Proc. Natl. Acad. Sci. U.S.A. 103:4658-4662.

Loper, J. E., and Schroth, M. N. 1986. Influence of bacterial sources of indole-3-acetic acid biosynthetic on root elongation of sugar beet. Phytopathology 76:386-389.

López-Bucio, J., Campos-Cuevas, J. C., Hernández-Calderón, E., Velásquez-Becerra, C., Farías-Rodríguez, R., Macías-Rodríguez, L. I., and Valencia-Cantero, E. 2007. Bacillus megaterium rhizobacteria promote growth and alter root-system architecture through an auxin and ethylene-independent signaling mechanism in Arabidopsis thaliana. Mol. Plant-Microbe Interact. 20:207-217.

Mark, G. L., Dow, J. M., Kiely, P. D., Higgins, H., Haynes, J., Baysse, C. Abbas, A., Foley, T., Franks, A., Morrissey, J., and O'Gara, F. 2005. Transcriptome profiling of bacterial responses to root exudates identifies genes involved in microbe-plant interactions. Proc. Natl. Acad. Sci. U.S.A. 102:17454-17459.

Mathysse, A. G., Stretton, S., Dandie, C., McClure, N. C., and Goodman, A. E. 1996. Construction of GFP vectors for use in gram-negative bacteria other than Escherichia coli. FEMS (Fed. Eur. Microbiol. Soc.) Microbiol. Lett. 145:87-94.

Navarro, L., Dunoyer, P., Jay, F., Arnold, B., Dharmasiri, N., Estelle, M., Voinnet, O., and Jones, J. D. G. 2006. A plant miRNA contributes to antibacterial resistance by repressing auxin signaling. Science 312:436439.

Nowak, J., Asiedu, S. K., Lazarovits, G., Pillay, V., Stewart, A., Smith, C., and Liu, Z. 1995. Enhancement of in vitro growth and transplant stress tolerance of potato and vegetable plants co-cultured with a plant growth promoting rhizobacterium. Pages 173-180 in: Ecophysiology and Photosynthetic In Vitro Cultures. CEA, Aix-en-Provence, France.

Ortíz-Castro, R., Contreras-Cornejo, H. A., Macías-Rodríguez, L., and López-Bucio, J. 2009. The role of microbial signals in plant growth and development. Plant Signal. Behav. 4:701-712.

Patten, C. L., and Glick, B. R. 2002a. Regulation of indoleacetic acid production in Pseudomonas putida GR12-2 by tryptophan and the stationary-phase sigma factor RpoS. Can. J. Microbiol. 48:635-642.

Patten, C. L., and Glick, B. R. 2002b. Role of Pseudomonas putida indoleacetic acid in development of the host plant root system. Appl. Environ. Microbiol. 68:3795-3801.

Persello-Cartieaux, F., David, P., Sarrobert, C., Thibaud, M. C., Achouak, W., Robaglia, C., and Nussaume, L. 2001. Utilization of mutants to analyze the interaction between Arabidopsis thaliana and its naturally root-associated Pseudomonas. Planta 212:190-198.

Phi, Q. T., Park, Y. M., Ryu, C. M., Park, S. H., and Ghim, S. Y. 2008. Functional identification and expression of indole-3-pyruvate decarboxylase from Paenibacillus polymyxa E681. J. Microbiol. Biotechnol. 18:1235-1244.

Pillay, V. K., and Nowak, J. 1997. Inoculum density, temperature and genotype effects on epiphytic and endophytic colonization and in vitro growth promotion of tomato (Lycopersicon esculentum L.) by a pseudomonad bacterium. Can. J. Microbiol. 43:354-361.

Porra, R., Thompson, W., and Kriedmann, P. 1989. Determination of accurate extinction coefficients and simultaneous equations for assaying chlorophyll $\mathrm{a}$ and $\mathrm{b}$ extracted with four different solvents: Verification of the concentration of chlorophyll standards by atomic absorption spectroscopy. Biochem. Biophys. Acta 975:384-394.

Prinsen, E., Costacurta, A., Michiels, K., Vanderleyden, J., and Van Onckelen, H. 1993. Azospirillum brasilense indole-3-acetic acid biosynthesis: Evidence for a non-tryptophan dependent pathway. Mol. Plant-Microbe Interact. 6:609-615.

Quiñones, B., Dulla, G., and Lindow, S. E. 2005. Quorum sensing regulates exopolysaccharide production, motility, and virulence in Pseudomonas syringae. Mol. Plant-Microbe Interact. 18:682-693.

Ramey, B. E., Koutsoudis, M., von Bodman, S. B., and Fuqua, C. 2004. Biofilm formation in plant-microbe associations. Curr. Opin. Microbiol. 7:602-609.

Růzicka, K., Ljung, K., Vanneste, S., Podhorská, R., Beeckman, T., Friml, 
J., and Benková, E. 2007. Ethylene regulates root growth through effects on auxin biosynthesis and transport-dependent auxin distribution. Plant Cell 19:2197-2212.

Sessitsch, A., Coenye, T., Sturz, A. V., Vandamme, P., Ait Barka, E., Salles, J. F., Van Elsas, J. D., Faure, D., Reiter, B., Glick, B. R., WangPruski, G., and Nowak, J. 2005. Burkholderia phytofirmans sp. nov., a novel plant-associated bacterium with plant-beneficial properties. Int. J. Syst. Evol. Microbiol. 55:1187-1192.

Spaepen, S., Vanderleyden, J., and Remans, R. 2007. Indole-3-acetic acid in microbial and microorganism-plant signaling. FEMS (Fed. Eur. Microbiol. Soc.) Microbiol. Rev. 31:425-448.

Spaepen, S., Das, F., Luyten, E., Michiels, J., and Vanderleyden, J. 2009. Indole-3-acetic acid-regulated genes in Rhizobium etli CNPAF512. FEMS (Fed. Eur. Microbiol. Soc.) Microbiol. Lett. 291:195-200.

Steindler, L., Bertani, I., De Sordi, L., Schwager, S., Eberl, L., and Venturi, V. 2009. LasI/R and RhlI/R quorum sensing in a strain of Pseudomonas aeruginosa beneficial to plants. Appl. Environ. Microbiol. 75:5131-5140.

Strader, L. C., Chen, G. L., and Bartel, B. 2010. Ethylene directs auxin to control root cell expansion. Plant J. 64:874-884.

Suárez-Moreno, Z. R., Devescovi, G., Myers, M., Hallack, L., MendonçaPreviato, L., Caballero-Mellado, J., and Venturi, V. 2010. Commonalities and differences in regulation of $\mathrm{N}$-acyl homoserine lactone quorum sensing in the beneficial plant-associated Burkholderia species cluster. Appl. Environ. Microbiol. 76:4302-4317.

Sun, Y., Cheng, Z., and Glick, B. R. 2009. The presence of a 1-aminocyclopropane-1-carboxylate (ACC) deaminase deletion mutation alters the physiology of the endophytic plant growth-promoting bacterium Burkholderia phytofirmans PsJN. FEMS (Fed. Eur. Microbiol. Soc.) Microbiol. Lett. 296:131-136.

Swarup, R., Perry, P., Hagenbeek, D., Van Der Straeten, D., Beemster, G. T., Sandberg, G., Bhalerao, R., Ljung, K., and Bennett, M. J. 2007. Ethylene upregulates auxin biosynthesis in Arabidopsis seedlings to enhance inhibition of root cell elongation. Plant Cell 19:2186-2196.

Theocharis, A., Bordiec, S., Fernandez, O., Paquis, S., Dhondt-Cordelier, S., Baillieul, F., Clément, C., and Barka, E. A. 2012. Burkholderia phytofirmans PsJN primes Vitis vinifera $\mathrm{L}$. and confers a better tolerance to low nonfreezing temperatures. Mol. Plant-Microbe Interact. 25:241-249.
Vadassery, J., Ritter, C., Venus, Y., Camehl, I., Varma, A., Shahollari, B. Novák, O., Strnad, M., Ludwig-Müller, J., and Oelmüller, R. 2008. The role of auxins and cytokinins in the mutualistic interaction between Arabidopsis and Piriformospora indica. Mol. Plant-Microbe Interact. 21:1371-1383.

Vande Broek, A., Lambrecht, M., Eggermont, K., and Vanderleyden, J. 1999. Auxins upregulate expression of the indole-3-pyruvate decarboxylase gene in Azospirillum brasilense. J. Bacteriol. 181:1338-1342.

Vande Broek, A., Gysegom, P., Ona, O., Hendrickx, N., Prinsen, E., Van Impe, J., and Vanderleyden, J. 2005. Transcriptional analysis of the Azospirillum brasilense indole-3-pyruvate decarboxylase gene and identification of a cis-acting sequence involved in auxin responsive expression. Mol. Plant-Microbe Interact. 18:311-323.

Van Puyvelde, S., Cloots, L., Engelen, K., Das, F., Marchal, K., Vanderleyden, J., and Spaepen, S. 2011. Transcriptome analysis of the rhizosphere bacterium Azospirillum brasilense reveals an extensive auxin response. Microb. Ecol. 61:723-728.

Vidal, E. A., Araus, V., Lu, C., Parry, G., Green, P. J., Coruzzi, G. M., and Gutiérrez, R. A. 2010. Nitrate-responsive miR393/AFB3 regulatory module controls root system architecture in Arabidopsis thaliana. Proc. Natl. Acad. Sci. U.S.A. 107:4477-4482.

Weilharter, A., Mitter, B., Shin, M. V., Chain, P. S., Nowak, J., and Sessitsch, A. 2011. Complete genome sequence of the plant growth-promoting endophyte Burkholderia phytofirmans strain PsJN. J. Bacteriol. 193:33833384.

Whitehead, N., Barnard, A., Slater, H., Simpson, N., and Salmond, G. 2001. Quorum-sensing in Gram-negative bacteria. FEMS (Fed. Eur. Microbiol. Soc.) Microbiol. Rev. 25:365-404.

Yang, S., Zhang, Q., Guo, J., Charkowski, A. O., Glick, B. R., Ibekwe, A. M., Cooksey, D. A., and Yang, C. H. 2007. Global effect of indole-3acetic acid biosynthesis on multiple virulence factors of Erwinia chrysanthemi 3937. Appl. Environ. Microbiol. 73:1079-1088.

Yuan, Z., Haudecoeur, E., Faure, D., Kerr, K., and Nester, E. 2008. Comparative transcriptome analysis of Agrobacterium tumefaciens in response to plant signal salicylic acid, indole-3-acetic acid and gammaamino butyric acid reveals signaling cross talk and Agrobacterium-plant co-evolution. Cell Microbiol. 10:2339-2354. 